Págs. 287-300

\title{
El rol de la unidad de bienestar estudiantil en la Universidad Estatal del Sur de Manabíl
}

\author{
The Role of Student Welfare at the Universidad Estatal del Sur de Manabí \\ Leopoldo Vinicio Venegas Loor, Paola Yadira Moreira Aguayo y Danny Eduardo Chávez Pozo²
}

\begin{abstract}
Resumen
Las Instituciones de Educación Superior (IES) en Ecuador han atravesado cambios muy profundos, históricamente desde la creación de la nueva Constitución del Ecuador del año 2008; dichos cambios han permitido que la universidad ecuatoriana fortalezca los principales procesos sustantivos como la vinculación, docencia e investigación, sin dejar de lado las actividades de gestión que son fundamentales para el óptimo desarrollo de lo antes nombrado. A estos procesos ya existentes se ha agregado un elemento clave, el bienestar estudiantil, que permite demostrar el interés que tienen las IES frente a los profesionales en formación. La presente investigación se desarrolla en la Universidad Estatal del Sur de Manabí (UNESUM), siendo su principal objetivo determinar la importancia y los servicios que oferta la unidad de bienestar estudiantil, la metodología utilizada fue de tipo exploratoria y descriptiva, donde se obtuvo información clave para determinar dentro de los principales resultados el mejorar los procesos apuntando hacia la calidad, brindar atención especializada a la comunidad educativa, incremento en la asignación de becas por los distintos componentes que oferta la IES, concluyendo en que la Unidad de Bienestar Estudiantil de la UNESUM es una dependencia significativa e importante, ya que atiende a toda la población estudiantil, y por ende debe de brindar calidad y calidez en sus servicios y procesos.
\end{abstract}

\section{Palabras clave}

Bienestar estudiantil, educación superior, universidad.

\section{Abstract}

Institutions of higher education (IES) in Ecuador have gone through very profound changes, historically since the creation of the new Constitution of Ecuador in 2008; These changes have allowed the Ecuadorian University strengthen main substantive processes as bonding, teaching and research, without neglecting the management activities which are essential for optimal development of the before appointed. These existing processes has been added a key element, the student welfare, which allows to demonstrate the interest with the IES to professionals in training. This research is carried out at the State University of the South of Manabi (UNESUM), being its main objective to determine the importance and impact of student welfare, the methodology used was exploratory and descriptive, where he earned key information to determine within the main results improving processes aiming towards quality, provide specialized care to the educational community, increased the allocation of scholarships for the different components that offer the IES, concluding that the UNESUM student welfare unit is a significant and important, dependence since it caters to the entire student population, and therefore you must provide quality and warmth in its services and processes.

\section{Keywords}

Student welfare, higher education, University.

\section{Cómo citar/Citation}

Venegas Loor, Leopoldo Vinicio; Moreira Aguayo, Paola Yadira; Chávez Pozo, Danny Eduardo (2019). El rol de la unidad de bienestar estudiantil en la Universidad Estatal del Sur de Manabí. Revista de Sociología de la Educación-RASE, 12 (2), 287-300. http://dx.doi.org/10.7203/ RASE.12.2.13916.

1 Este artículo forma parte del proyecto TO-INN From Tradition to Innovation in Teacher Training Institutions financiado por la Unión Europea a través del programa Erasmus, Acción K2 Capacity Building, (573685-EPP-2016-1-ES-EPPKA2-CBHE-JP) https://www.toinn.org.

2 Leopoldo Vinicio Venegas Loor, Universidad Estatal del Sur de Manabí, leopoldo.venegas@unesum.edu.ec; Paola Yadira Moreira Aguayo, Universidad Estatal del Sur de Manabí, paola.moreira@unesum.edu.ec; Danny Eduardo Chávez Pozo, chavezdanny1@hotmail.com. 


\section{Introducción}

De conformidad a lo que establece la Ley Orgánica de Educación Superior (LOES), en su artículo 86. -Unidad de bienestar estudiantil-. Las instituciones de educación superior mantendrán una unidad administrativa de Bienestar Estudiantil destinada a promover la orientación vocacional y profesional, facilitar la obtención de créditos, estímulos, ayudas económicas y becas, y ofrecer los servicios asistenciales que se determinen en las normativas de cada institución. Esta unidad, además, se encargará de promover un ambiente de respeto a los derechos y a la integridad física, psicológica y sexual de las y los estudiantes, en un ambiente libre de violencia, y brindará asistencia a quienes demanden por violaciones de estos derechos. La Unidad de Bienestar Estudiantil de cada institución formulará e implementará políticas, programas y proyectos para la prevención y atención emergente a las víctimas de delitos sexuales, además de presentar, por intermedio de los representantes legales, la denuncia de dichos hechos a las instancias administrativas y judiciales según la Ley. Se implementarán programas y proyectos de información y prevención integral del uso de drogas, bebidas alcohólicas, cigarrillos y derivados del tabaco, y coordinará con los organismos competentes para el tratamiento y rehabilitación de las adicciones en el marco del plan nacional sobre drogas. (CES, 2010)

El propósito de la creación de las Unidades de Bienestar Estudiantil forma parte del plan estratégico que tienen las universidades para contribuir con el mejoramiento de la calidad académica de los estudiantes; por ende, su creación e implementación se convierte en elemento fundamental e instrumental para las IES, constituyéndose los servicios que proporcionan garantías del bienestar estudiantil lo cual se suma al nivel intelectual, social y profesional.

La zona sur de Manabí cuenta con la UNESUM destinada a formar profesionales calificados, competentes, emprendedores, innovadores, críticos y comprometidos con el desarrollo de diversas áreas del conocimiento para mejorar la calidad de vida de la población.

El presente trabajo se desarrolla con la finalidad de dar a conocer los diferentes servicios que brinda o presta la unidad de bienestar estudiantil de las UNESUM y su importancia para los profesionales en formación.

\subsection{El Rol de la Universidad}

La universidad es el elemento principal para el progreso de nuestra sociedad y economía, es el ente encargado de generar conocimiento, que es sinónimo de riqueza, bienestar y desarrollo, permitiendo así la igualdad de oportunidades para la población y un ascenso social en la comunidad. Las funciones, tareas y objetivos de la academia han ido incrementándose con el transcurso del tiempo, ha esto se ha sumado factores como la innovación, la creatividad, y la calidad; y algo fundamental que permite una verdadera sintaxis en la educación superior son los centros de investigación continúa, lo cual asegura una educación superior de calidad (Rodríguez-Ponce, 2009).

De esta realidad no está alejada la UNESUM, donde se enfoca en fortalecer los tres procesos sustantivos como lo son la Docencia, Investigación y Vinculación con la Sociedad, generando proyectos, investigaciones y resultados que aportan al desarrollo social y equitativo de la población de la zona sur de Manabí.

\subsection{El Bienestar Estudiantil Universitario en Ecuador}

En este punto vale resaltar una breve historia de las Unidades de Bienestar Estudiantil en Ecuador; 
estas surgen mediante mandato constitucional. La Constitución de la República del Ecuador aprobada en Montecristi en el año 2008, da mucho énfasis a la educación superior, considerándola universal y gratuita, además se indica que será de interés público, y que mediante ella se impulsara la equidad de género, la justicia, la solidaridad y la paz; estimulara el sentido crítico, el arte y la cultura física, la iniciativa individual y comunitaria y el desarrollo de competencias y capacidades para crear y trabajar, que promueva el dialogo intercultural en todas sus dimensiones (Asamblea Nacional Constituyente, 2008b)

Básicamente los Art. 27 y 28 de la Constitución se consideran el génesis del bienestar estudiantil universitario, algo que anteriormente se lo hacía, pero de manera mínima, sencilla y en muchos casos aislada. Es entonces a raíz de la carta magna que se da énfasis al bienestar estudiantil; a medida que se evolucionaba en perfeccionar el sistema educativo universitario del Ecuador, más adelante se crearon leyes que en su cuerpo incluían la importancia y los objetivos del bienestar estudiantil.

\subsection{Unidad de Bienestar Estudiantil UNESUM}

La Unidad de Bienestar estudiantil (UBE) de la Universidad Estatal del Sur de Manabí, se constituye en una unidad académica dependiente de la Dirección General Académica, cumpliendo lo que establece la Ley Orgánica de Educación Superior del Ecuador (2010); este departamento de la UNESUM es uno de sus pilares estratégicos y de gestión social de esta universidad y a la vez se parte de la Responsabilidad Social Universitaria.

La unidad de bienestar estudiantil de la UNESUM consta según estatuto (2015) de la UNESUM, en el capítulo V, Art. 74. "La Universidad Estatal del Sur de Manabi mantendrá una Unidad Administrativa de Bienestar Estudiantil, destinada a promover la orientación vocacional y profesional, facilitar la obtención de créditos, estímulos, ayudas económicas y becas, y ofrecer los servicios asistenciales que se determinen en las normativas internas». (Universidad Estatal del Sur de Manabí, 2015: 35).

La Unidad de Bienestar estudiantil nació con el propósito de prestar servicios, becas y ayudas económicas a los y las estudiantes de la comunidad universitaria. A partir de la administración del actual rector PhD. Omelio Borroto Leal, donde surgen cambios muy importantes en la Unidad de Bienestar Estudiantil, mejorando su infraestructura e incrementando servicios, para antender de una mejor manera a la comunidad educativa.

Para la comunidad educativa esta unidad es fundamental, ya que representa el nexo entre los diferentes niveles de atención en la institución, reciben apoyo sicológico, procesos para un acompañamiento personalizado en conjunto con los tutores de cada carrera, charlas motivacionales, planificación familiar, entre otros que esta unidad brinda día a día a la institución. El contar con esta unidad, da un enfoque positivo al desarrollo de la UNESUM, y gracias a esto garantizan el desarrollo de las capacidades colectivas e individuales que permitan el mejoramiento de la calidad de vida de la comunidad educativa.

\subsection{De las becas y ayudas económicas en la UNESUM}

Según el Estatuto de la UNESUM en su Art. 98. La Universidad Estatal del Sur de Manabí establecerá programas de becas completas o su equivalente en ayudas económicas, que apoyen en su escolaridad a por lo menos el 10\% del número de estudiantes regulares. Serán beneficiados los/as estudiantes regulares con alto promedio y distinción académica, los/as estudiantes que no cuenten con recursos 
económicos suficientes, los/as discapacitados/as, los/as deportistas de alto rendimiento que representen al país en eventos internacionales, a condición de que acrediten niveles de rendimiento académicos conforme la reglamentación institucional respectiva. (Universidad Estatal del Sur de Manabí, 2015: 44).

\subsection{Objetivos de la Unidad de Bienestar Estudiantil UNESUM}

Según lo establecido en el reglamento de la Unidad de Bienestar Estudiantil (Universidad Estatal del Sur de Manabí, 2015: 4), son objetivos los siguientes:

a) Monitorear la calidad de los servicios que ofrece la institución en beneficio de los/as estudiantes, a través de encuestas, observación directa y entrevistas;

b) Ofrecer información y asesoría a los estudiantes a fin de facilitar el conocimiento referente a sus derechos y obligaciones como estudiantes universitarios, mediante la atención directa, visita a las aulas, informativos impresos y página web institucional;

c) Gestionar convenios, becas para brindar oportunidades de perfeccionamiento y continuidad de estudios a los alumnos en cada ámbito de estudio;

d) Promover un ambiente de respeto a los derechos y a la integridad física, psicología y sexual de los estudiantes en un ambiente libre de violencia;

e) Propiciar soluciones a los problemas psicopedagógicos de los estudiantes en coordinación con las facultades;

f) Atender los reclamos de los estudiantes, dar solución y garantiza la no retaliación, mediante la implementación de un sistema de comunicación de reclamos y la coordinación con los organismos estudiantiles y facultades;

g) Evaluar el grado de satisfacción de los estudiantes con respecto a la resolución de reclamos y la oferta de servicios en general, mediante entrevistas y encuestas;

h) Implementar programas y proyectos de información y prevención integral del uso de drogas, bebidas alcohólicas, cigarrillos y derivados del tabaco.

\subsection{Misión de la Unidad de Bienestar Estudiantil UNESUM}

Gestionar programa y proyectos que contribuyan al bienestar de todos/as los/as estudiantes, fomentando una cultura colectiva de desarrollo humano a través de programas de salud integral, trabajo social, orientación vocacional, psicológica, becas y ayudas económicas, recreación y otros. (Universidad Estatal del Sur de Manabí, 2015: 2).

\subsection{Visión de la Unidad de Bienestar Estudiantil UNESUM}

Contribuir a la generación, implementación, y seguimiento de actividades que permiten mejorar las condiciones en las que se desenvuelven los/as estudiantes. (Universidad Estatal del Sur de Manabí, 2015: 2). 


\subsection{Servicios que oferta la Unidad de Bienestar Estudiantil UNESUM}

Tabla I. Servicios de la UBE

\begin{tabular}{lc}
\hline \multicolumn{1}{c}{ SERVICIOS } & PROFESIONAL ENCARGADO \\
\hline $\begin{array}{l}\text { Coordinación del Área. Se encarga de gestionar los diferentes procesos de la unidad, con el } \\
\text { fin de brindar servicios de calidad y optimizar los procesos de la misma. }\end{array}$ & Coordinador/a del área \\
\hline Médico. Se encarga de brindar los servicios de salud a toda la comunidad educativa. & Médico General \\
\hline $\begin{array}{l}\text { Odontológico. Se encarga de brindar los servicios de salud bucal a toda la comunidad } \\
\text { educativa. }\end{array}$ & Odontólogo/a \\
\hline $\begin{array}{l}\text { Orientación Vocacional. Se encarga de realizar análisis de la realidad educativa y los } \\
\text { factores socioculturales que influyen en la formación profesional de los estudiantes a fin de } \\
\text { conocer los aspectos que favorecen o afectan el proceso docente. }\end{array}$ & Psicólogo/a Educativo \\
\hline \begin{tabular}{l} 
Psicología Clínica \\
\hline Trabajo Social. Se encarga de realizar las verificaciones de la situación socioeconómica \\
de los estudiantes que soliciten becas y ayudas económicas, y emitir los informes cor-
\end{tabular} & Psicólogo/a Clínico \\
respondientes. & Trabajador/a Social \\
\hline $\begin{array}{l}\text { Becas. Producto que se brinda a los estudiantes como apoyo para la continuidad de sus } \\
\text { estudios, donde existen los componentes de Rendimiento Académico, Situación Socioec- } \\
\text { onómica, Discapacidad, Cultura y Deporte. }\end{array}$ & Coordinador/a del área \\
\hline $\begin{array}{l}\text { Control de bares y puntos de expendio. Se encargan de dar seguimiento al cumplimiento de } \\
\text { normativas de calidad e higiene, así como el poseer la documentación reglamentaria al día. }\end{array}$ & Secretaria \\
\hline
\end{tabular}

Fuente: Reglamento General de la Unidad de Bienestar Estudiantil UNESUM, https://drive.google.com/file/ d/0B1yqOTYPrKWPQzBqUGZ6Y0ZmdUU/view.

Elaborado por: Ing. Leopoldo Venegas Loor, Mg. Eas.

\section{Materiales y métodos}

Esta investigación es de tipo exploratoria y descriptiva, por cuanto pretende brindar información de los servicios que oferta la Unidad de Bienestar Estudiantil de la Universidad Estatal del Sur de Manabí, en base al cumplimiento de lo que establece el Artículo 74 antes descrito del Estatuto UNESUM, realizando un autodiagnóstico que permita a la universidad conocer cuál es el papel fundamental que desarrolla la UBE en la UNESUM.

El trabajo de campo se desarrolló en la Universidad Estatal del Sur de Manabí en base a la información que tiene la Unidad de Bienestar Estudiantil.

\section{Resultados}

De la información obtenida en la Unidad de Bienestar Estudiantil, se pudo evidenciar los siguientes resultados tomando en consideración de Enero - Septiembre 2018.

\subsection{Proyectos y programas ejecutados en el 2018}

A continuación, se detalla cada uno de los programas con el que cuenta la Unidad de Bienestar Estudiantil de la UNESUM, con el fin de dar a conocer los resultados obtenidos en la ejecución de cada uno de ellos.

Tal como lo establece el reglamento de la Unidad de Bienestar Estudiantil de la UNESUM en el Art. 5, que son políticas de esta formular e implementar programas y proyectos en el área de la salud, orientación, prevención, entre otros, (Universidad Estatal del Sur de Manabí, 2016: 3). 
Tabla II. Programas y Proyectos de la UBE

\begin{tabular}{|c|c|c|c|}
\hline N. ${ }^{\circ}$ & PROYECTO Y/O PROGRAMA & ACTIVIDADES REALIZADAS & MES/2018 \\
\hline 1 & Plan de ética & $\begin{array}{c}\text { Socialización de los reglamentos de ética y acción afirma- } \\
\text { tiva por carreras - UNESUM }\end{array}$ & Enero \\
\hline 2 & Proyecto alcohol, tabaco y drogas & $\begin{array}{l}\text { Socialización del Acuerdo de prevención Integral de drogas } \\
\text { con instituciones de educación superior } \\
\text { Conferencia sobre Prevención integral del uso de alcohol, } \\
\text { tabaco y droga. }\end{array}$ & Febrero - Julio - Agosto \\
\hline 3 & $\begin{array}{l}\text { Programa de orientacion, acompañamiento y permanencia } \\
\text { de los estudiantes de la unesum }\end{array}$ & $\begin{array}{l}\text { Capacitación a los docentes de nivelación en el uso de } \\
\text { herramientas pedagógicas para atender las necesidades } \\
\text { educativas de la discapacidad que posee el estudiante no } \\
\text { vidente (Fundación Oswaldo Loor - UNESUM) } \\
\text { Asesoría de mecanismos inclusivos dentro del aula, como } \\
\text { la digitalización de los textos a utilizar para que puedan } \\
\text { portarse en el móvil y usarlos con el lector de pantalla. }\end{array}$ & Mayo \\
\hline \multirow{3}{*}{4} & \multirow{3}{*}{ Proyecto integral de salud sexual para jovenes } & $\begin{array}{l}\text { Campaña de donación de medicamentos para habilitar } \\
\text { botiquín de emergencia para la comunidad universitaria - } \\
\text { UNESUM } 2018\end{array}$ & Enero \\
\hline & & $\begin{array}{l}\text { Conferencia «Sexualidad responsable en la formación } \\
\text { universitaria» (estudiantes de Nivelación) }\end{array}$ & Marzo - Julio \\
\hline & & Charla educativa sobre OBESIDAD, Nutricion y loERRZGO & Agosto \\
\hline
\end{tabular}

Fuente: Unidad de Bienestar Estudiantil UNESUM

Elaborado por: Ing. Leopoldo Venegas Loor, Mg. Eas.

\subsection{Plan de promoción de comportamiento ético y prevención del comportamiento antiético}

Socialización del plan de promoción de comportamiento ético y prevención del comportamiento antiético a los miembros de la comunidad universitaria de la UNESUM, con el objetivo de que los estudiantes se rijan bajo las normativas que establece la institución, y no cometan actos que atenten contra los principios de convivencia universitaria, ni cometan actos que deshonren el nombre institucional, en este plan que se llevó a cabo en conjunto con el personal de bienestar universitario en donde participaron 1190 profesionales en formación.

\section{Gráfico 1. Plan de promoción de comportamiento ético y prevención del comportamiento antiético}

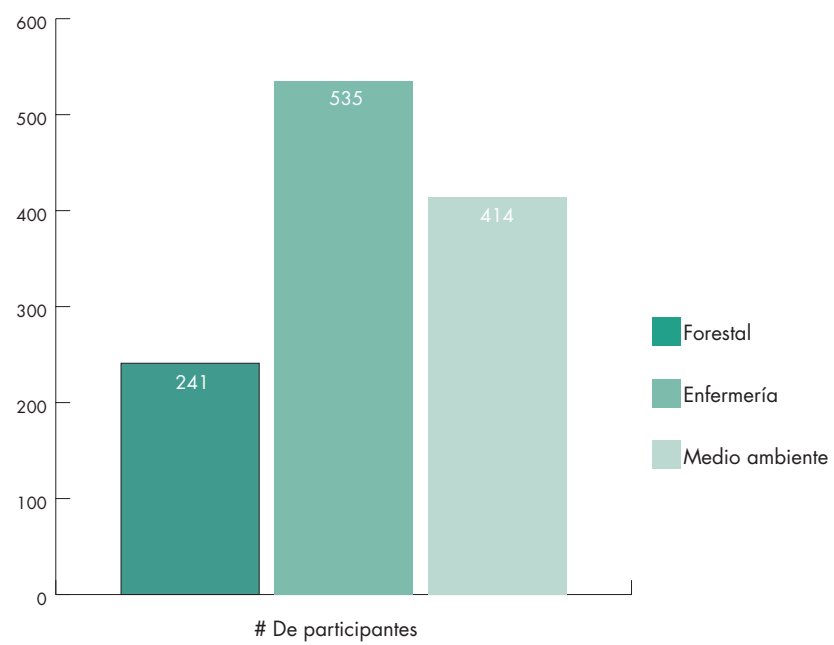

Fuente: Unidad de Bienestar Estudiantil UNESUM. 


\subsection{Proyecto alcohol, tabaco y drogas}

Este proyecto está enfocado en crear una cultura de prevención en toda la comunidad universitaria, con el fin de disminuir los niveles de consumo detectados por el área médica y trabajo social de la Unidad de Bienestar Estudiantil.

Tabla III. Conferencia sobre prevención integral del uso de alcohol, tabaco y droga

\begin{tabular}{|c|c|c|c|c|c|c|}
\hline \multirow{3}{*}{ FACULTAD } & \multirow{3}{*}{ CARRERA } & \multirow{2}{*}{\multicolumn{3}{|c|}{$\begin{array}{c}\text { NÚMERO DE ESTUDIANTES POR CARRERA } \\
\text { ENERO - SEPTIEMBRE } 2018\end{array}$}} & \multirow{3}{*}{ TOTAL } & \multirow{3}{*}{ PORCENTAJE } \\
\hline & & & & & & \\
\hline & & FEBRERO & JULIO & AGOSTO & & \\
\hline \multirow{4}{*}{ Ciencias Técnicas } & Tecnología de la información & 37 & 64 & - & 101 & $11 \%$ \\
\hline & Computación y redes & 38 & 10 & 8 & 56 & $6 \%$ \\
\hline & Sistemas computacionales & 0 & 29 & - & 29 & $3 \%$ \\
\hline & Ingeniería civil & 56 & 140 & 72 & 268 & $29 \%$ \\
\hline \multirow{3}{*}{ Ciencias Naturales y Agropecuarias } & Agropecuaria & 0 & 0 & - & 0 & $0 \%$ \\
\hline & Forestal & 0 & 0 & - & 0 & $0 \%$ \\
\hline & Medio Ambiente & 36 & 67 & - & 103 & $11 \%$ \\
\hline \multirow{6}{*}{ Ciencias Administrativas y Económicas } & Comercio Exterior & 0 & 11 & 7 & 18 & $2 \%$ \\
\hline & Auditoría & 59 & 18 & - & 77 & $8 \%$ \\
\hline & Gestión Empresarial & 0 & 15 & - & 15 & $2 \%$ \\
\hline & Turismo & 0 & 19 & 11 & 30 & $3 \%$ \\
\hline & Adm. Emp. Agropecuarias & 0 & 0 & 19 & 19 & $2 \%$ \\
\hline & Administración & 0 & 0 & - & 0 & $0 \%$ \\
\hline \multirow{2}{*}{ Ciencias de la Salud } & Enfermería & 30 & 0 & 57 & 87 & $9 \%$ \\
\hline & Laboratorio clínico & 31 & 88 & - & 119 & $13 \%$ \\
\hline Total & & 287 & 461 & 174 & 922 & $100 \%$ \\
\hline
\end{tabular}

Fuente: Unidad de Bienestar Estudiantil UNESUM.

Elaborado por: Ing. Leopoldo Venegas Loor, Mg. Eas.

Del número de participantes, hay carreras que lamentablemente no apoyan a la ejecución de este tipo de proyecto, pese a que son invitados a participar del mismo, lo que conlleva que no se pueda abarcar a una gran cantidad de la población estudiantil.

\section{4. Área de servicio asistencial}

El reglamento de la Unidad de Bienestar Estudiantil, establece los servicios asistenciales, dentro del cual establece que (Universidad Estatal del Sur de Manabí, 2016: 6-7):

Art. 10. Funciones del Médico:

a) Programar los servicios de salud dentro de la UNESUM;

b) Elaborar y mantener las historias clínicas (Fichas médicas del estudiante - paciente);

c) Realizar los reportes y estadísticas de los pacientes atendidos de manera mensual;

d) Promover y mantener charlas y proyectos que propicien un ambiente de respeto a los valores éticos y la integridad física y sexual de las y los estudiantes; 
e) Remitir y canalizar las peticiones referentes a medicamentos, insumos, equipamiento e implementación de esta área;

f) Cumplir con el horario establecido de acuerdo con los requerimientos de la UNESUM; y,

g) Cumplir de acuerdo a las demás que determine la Ley, el Estatuto y los Reglamentos.

Art. 11. Funciones del Odontólogo:

a) Programar los servicios de salud bucal dentro de la Universidad;

b) Presentar y ejecutar proyectos y programas preventivos y correctivos de higiene bucal;

c) Elaborar y mantener las historias clínicas (Fichas Odontológica del estudiante - paciente);

d) Realizar los reportes y estadísticas de los pacientes atendidos de manera mensual;

e) Remitir y canalizar las peticiones referentes a medicamentos, insumos, equipamiento e implementación de esta área;

f) Cumplir con el horario establecido de acuerdo con los requerimientos de la Universidad; y,

g) Cumplir de acuerdo a las demás que determine la Ley, el Estatuto y los Reglamentos.

En los meses de enero a septiembre/2018 recibieron atención médica y odontológica un total de 2299 miembros de la comunidad universitaria, como consta a continuación:

Tabla IV. Servicios asistenciales

\begin{tabular}{lccc}
\hline N. & MES & & ÁREA \\
\hline 1 & & MÉDICA & ODONTOLÓGICA \\
\hline 2 & Enero & 120 & 48 \\
\hline 3 & Febrero & 137 & 20 \\
\hline 4 & Marzo & 126 & 9 \\
\hline 5 & Abril & 393 & 182 \\
\hline 6 & Mayo & 260 & 120 \\
\hline 7 & Junio & 300 & 60 \\
\hline 8 & Julio & 218 & 37 \\
\hline 9 & Agosto & 140 & 39 \\
\hline Total & Septiembre & 60 & 30 \\
\hline
\end{tabular}

Fuente: Unidad de Bienestar Estudiantil UNESUM.

Elaborado por: Ing. Leopoldo Venegas Loor, Mg. Eas. 


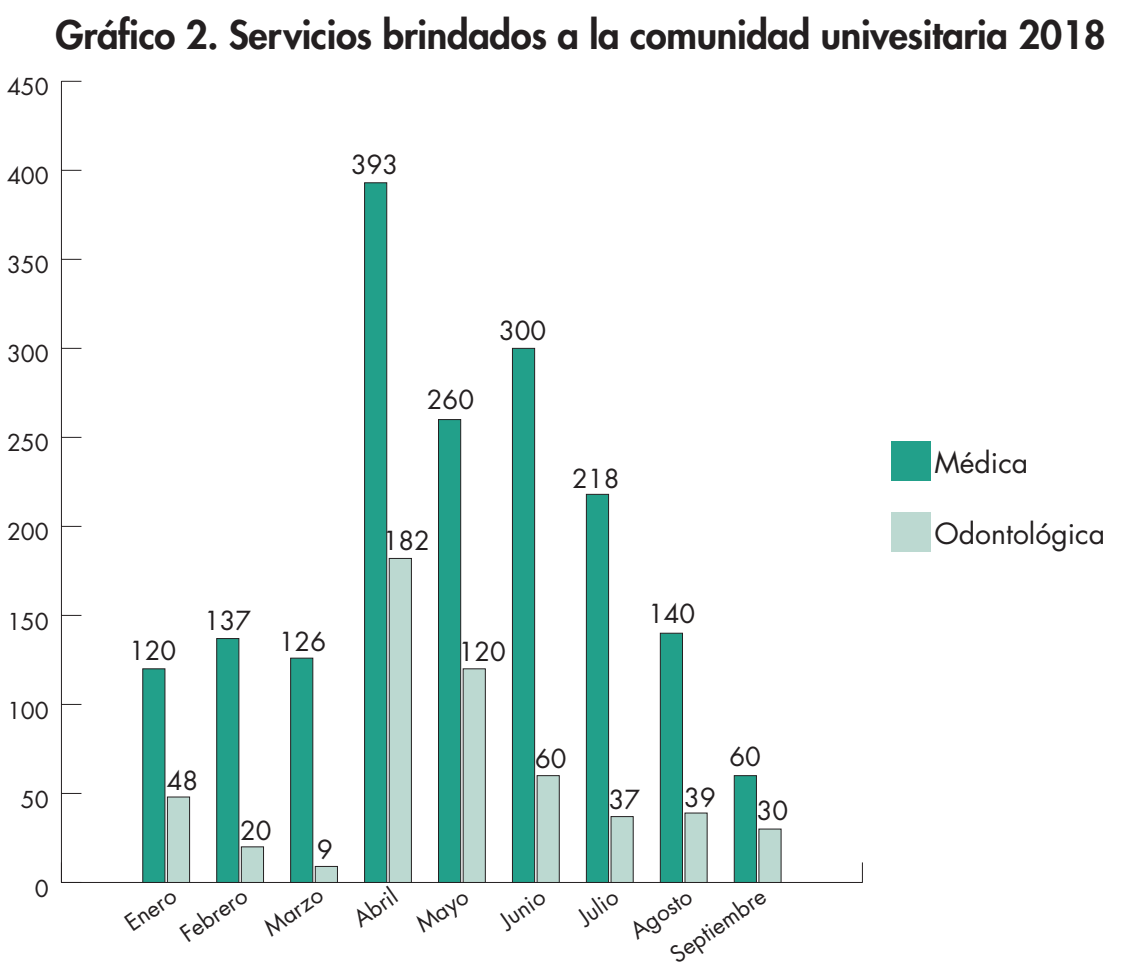

Fuente: Unidad de Bienestar Estudiantil UNESUM.

\subsection{Becas y ayudas económicas UNESUM}

Tal como lo establece el Art. 3 del Reglamento de Becas y Ayudas Económicas para estudiantes de pregrado de la UNESUM, las becas y ayudas económicas estarán coordinadas y supervisadas por la Unidad de Bienestar Estudiantil, conforme lo que establece la Ley Orgánica de Educación Superior, quien emitirá el informe respectivo a la comisión calificadora de becas y ayudas económicas, para su respectiva adjudicación, (Universidad Estatal del Sur de Manabí, 2019: 2-3).

Para lo cual se definen ocho clases de becas estipulado en el Art. 9 (Universidad Estatal del Sur de Manabí, 2019: 4-5):

a. Becas por resultados académicos: Son becas económicas, adjudicadas a los/as estudiantes de alto rendimiento académico, que obtuvieron en el periodo académico anterior a la convocatoria de becas, el mejor promedio por nivel o semestre paralelo de cada carrera. Con un promedio mínimo de nueve sobre diez puntos $(9,00 / 10)$

b. Becas por situación socio-económica: Son becas económicas, adjudicadas a estudiantes de limitados recursos económicos, pertenecientes a minorías étnicas, solidarias y comunidades históricamente relegadas, que obtuvieron en el período académico anterior a la convocatoria de becas, un promedio mínimo de siete con cincuenta sobre diez puntos $(7,50 / 10)$.

c. Becas por movilidad territorial: Son becas económicas, adjudicadas a estudiantes de limitados recursos económicos que por asignación del cupo del SNNA debieron cambiar su residencia habitual a la ciudad de Jipijapa, que obtuvieron en el período académico anterior a la convocatoria de becas, un promedio mínimo de ocho sobre diez puntos (8,00/10).

d. Becas por resultados en participación en concursos académicos: Son becas económicas, adjudicadas a estudiantes triunfadores de los 3 primeros lugares en concursos nacionales e internacio- 
nales en el área académica, que obtuvieron en el periodo académico anterior a la convocatoria de becas, un promedio mínimo de ocho con cincuenta sobre diez puntos $(8,50 / 10)$.

e. Becas culturales: Son becas económicas, adjudicadas a los estudiantes destacados en las distintas áreas artístico - culturales a nivel nacional e internacional, coordinado por el Área de Cultura previo a la aprobación del Vicerrectorado Académico, que obtuvieron en el periodo académico anterior a la convocatoria de becas, un promedio mínimo de ocho con cincuenta sobre diez puntos $(8,50 / 10)$.

f. Becas por investigación: Son becas económicas, adjudicadas a los estudiantes, como reconocimiento al trabajo de investigación que haya contribuido al desarrollo institucional, este deberá ser analizado y avalado por la Dirección de Investigación y Posgrado, que obtuvieron en el periodo académico anterior a la convocatoria de becas, un promedio mínimo de ocho cincuenta sobre diez puntos $(8,50 / 10)$.

g. Becas deportivas: Son becas económicas, adjudicadas a los estudiantes destacados nacional o internacionalmente en las distintas disciplinas deportivas que han representado al país, que obtuvieron en el periodo académico anterior a la convocatoria de becas, un promedio mínimo de ocho cincuenta sobre diez puntos $(8,50 / 10)$. Los beneficiarios de esta beca deberán demostrar responsabilidad académica durante su permanencia en la universidad.

h. Becas por situación de discapacidades: Son becas económicas, adjudicadas a los estudiantes con discapacidades en condición permanente conforme las disposiciones y exigencias del Consejo Nacional de Discapacidades, que obtuvieron en el período académico anterior a la convocatoria de becas, un promedio mínimo de siete sobre diez puntos (7,00/10). Los beneficiarios de esta beca deberán demostrar responsabilidad académica durante su permanencia en la universidad.

Se adjudicaron BECAS a estudiantes de las diferentes carreras que oferta la UNESUM, respectivamente, tal como se refleja en la siguiente tabla:

Tabla V. Servicio de becas y ayudas económicas

\begin{tabular}{|c|c|c|c|}
\hline \multirow[t]{2}{*}{ N. ${ }^{\circ}$} & \multirow[t]{2}{*}{ CLASES DE BECAS } & \multicolumn{2}{|c|}{ \# DE BECARIOS } \\
\hline & & NOVIEMBRE 2017 - MARZO 2018 & MAYO - SEPTIEMBRE 2018 \\
\hline 1 & Cultural & 0 & 4 \\
\hline 2 & Damnificados por desastres naturales & 42 & 0 \\
\hline 3 & Resultados académicos & 94 & 144 \\
\hline 4 & Resultados académicos y situación socio-económica & 12 & 108 \\
\hline 5 & Resultados académicos y movilidad territorial & 27 & 87 \\
\hline 6 & Situación de discapacidades & 5 & 4 \\
\hline Total & & 180 & 347 \\
\hline
\end{tabular}

Fuente: Unidad de Bienestar Estudiantil UNESUM.

Elaborado por: Ing. Leopoldo Venegas Loor, Mg. Eas. 


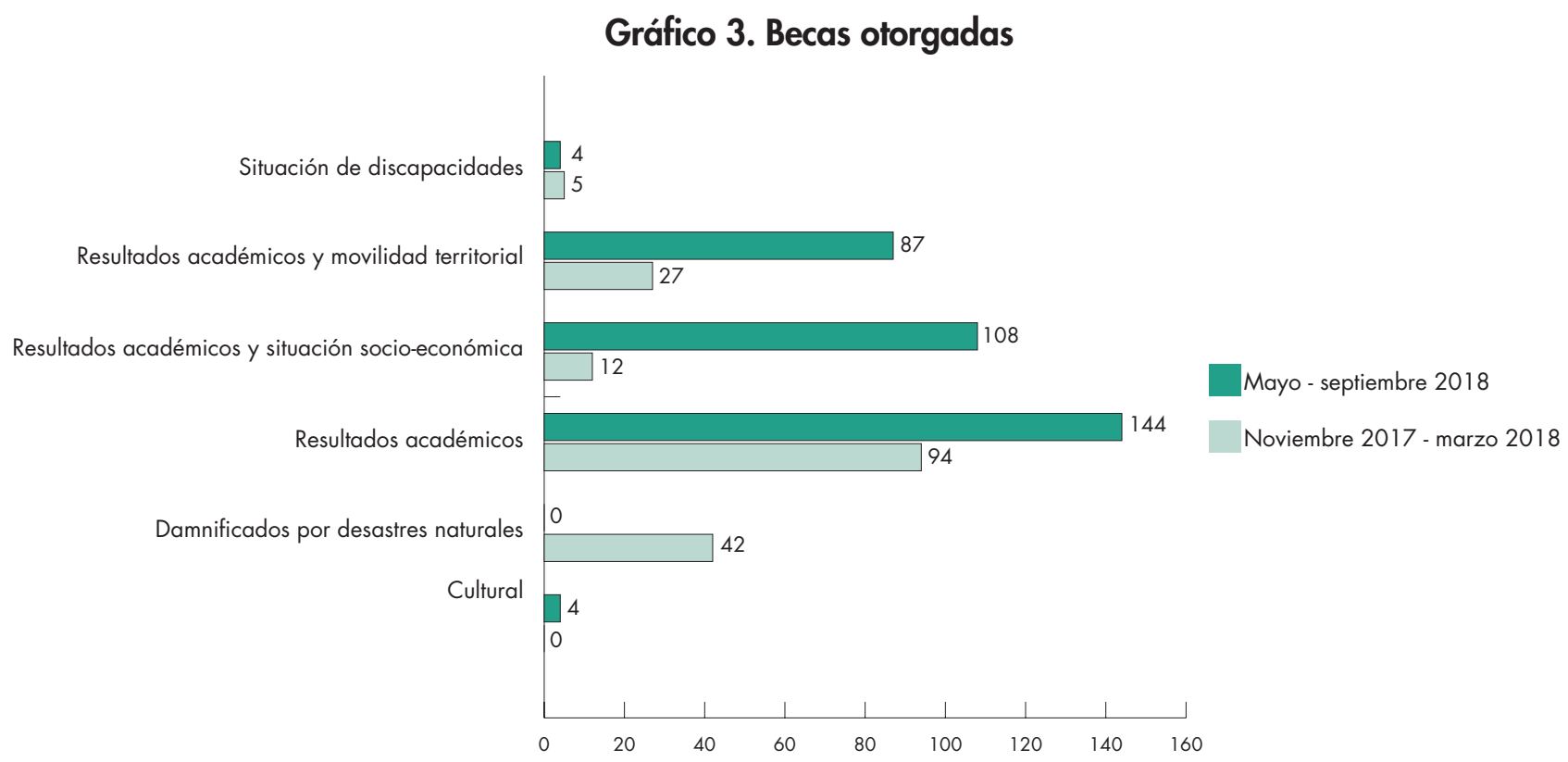

Fuente: Unidad de Bienestar Estudiantil UNESUM.

\subsection{Ayudas económicas}

Son ayudas económicas según el Art. 20 del Reglamento de Becas y Ayudas Económicas para estudiantes de grado, lo siguiente (Universidad Estatal del Sur de Manabí, 2019: 7):

Art. 20. Ayuda Económica. - Constituye un apoyo monetario de carácter excepcional y específico no reembolsable, orientado a financiar, por una sola vez y de forma emergente, con fines académicos, a estudiantes del sistema de educación superior, con el objeto de cubrir el costo de rubros inherentes a su formación superior, capacitación, perfeccionamiento, entrenamiento y/o programas de investigación, de conformidad a la normativa vigente.

Art. 21. Clases de Ayudas Económicas. - Las ayudas económicas serán otorgadas como apoyo al desarrollo académico e investigativo de los estudiantes y son:

a. Capacitación: Son ayudas económicas a estudiantes para financiar actividades de capacitación determinadas y aprobadas por la Institución.

b. Concursos: Son ayudas económicas a estudiantes que participen en concursos locales, nacionales e internacionales en representación de la Institución.

c. Investigación: Son ayudas económicas a estudiantes que participen en programas de investigación con resultados relevantes y representen a la Institución en eventos de este tipo.

Se adjudicaron Ayudas económicas a estudiantes de las diferentes carreras que oferta la UNESUM, respectivamente: 
Tabla VI. Ayudas económicas. Periodo Académico: Noviembre 2017 - marzo 2018

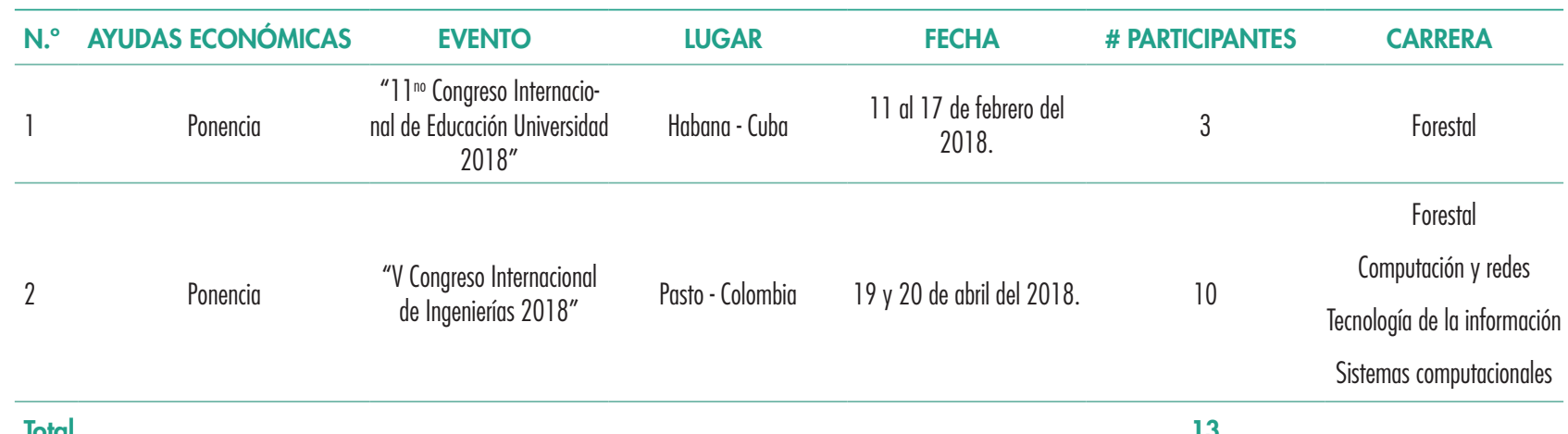

Fuente: Unidad de Bienestar Estudiantil UNESUM.

Elaborado por: Ing. Leopoldo Venegas Loor, Mg. Eas.

Tabla VII. Ayudas económicas. Periodo Académico: Mayo - septiembre 2018

\begin{tabular}{|c|c|c|c|c|c|c|}
\hline N. ${ }^{\circ}$ & AYUDAS ECONÓMICAS & EVENTO & LUGAR & FECHA & \# PARTICIPANTES & CARRERA \\
\hline 1 & Ponencia & $\begin{array}{l}\text { "VI Congreso la Sociedad } \\
\text { Cubana de Medicina } \\
\text { Bioenergética y Naturalista } \\
\text { BIONAT 2018" }\end{array}$ & Habana - Cuba & $\begin{array}{c}03 \text { al } 07 \text { de septiembre } \\
\text { del } 2018\end{array}$ & 1 & Enfermería \\
\hline Total & & & & & 1 & \\
\hline
\end{tabular}

Fuente: Unidad de Bienestar Estudiantil UNESUM.

Elaborado por: Ing. Leopoldo Venegas Loor, Mg. Eas.

\subsection{Asesoramiento técnico de los Programas de Becas Nacionales - Instituto de Fomento de Talento Humano}

El equipo técnico de la Unidad de Bienestar Estudiantil, realiza un proceso de asesoramiento para aquellos estudiantes que quieran postular a las becas ofrecidas por el Gobierno Nacional.

En los meses de enero a septiembre/2018 recibieron asesoría técnica un total de 410 miembros de la comunidad universitaria, como consta a continuación:

Tabla VIII. Asesoramiento técnico, Becas Instituto de Fomento de Talento Humano

\begin{tabular}{lcc}
\hline$N .^{\circ}$ & MES & \# DE ESTUDIANTES ATENDIDOS \\
\hline 1 & Enero & 5 \\
\hline 2 & Febrero & 13 \\
\hline 3 & Marzo & 69 \\
\hline 4 & Abril & 90 \\
\hline 5 & Mayo & 94 \\
\hline 6 & Junio & 18 \\
\hline 7 & Julio & 36 \\
\hline 8 & Agosto & 23 \\
\hline 9 & Septiembre & 62 \\
\hline Total & & 410 \\
\hline
\end{tabular}

Fuente: Unidad de Bienestar Estudiantil UNESUM.

Elaborado por: Ing. Leopoldo Venegas Loor, Mg. Eas. 


\section{Discusión}

Se evidencia una mejora en la oferta de los servicios por parte de la Unidad de Bienestar Estudiantil, estableciendo procesos de calidad hacia la comunidad universitaria, los servicios médicos y odontológicos se han tratado de mejorar en cuanto a la implementación de herramientas que permitan brindar mejores servicios, en el área asistencial de medicina general se implementó un grupo de medicamentos para poder atender las diversas enfermedades que presente la comunidad universitaria; a partir del periodo académico Noviembre 2017 - marzo 2018 llegó a la unidad un profesional en el área de sicología clínica, con el cual se comienza a desarrollar una serie de actividades importantes para atender de manera especializada a la comunidad educativa, brindando charlas, capacitaciones y trabajando conjuntamente con otras dependencias de la UNESUM, se hace seguimiento académico a los estudiantes adjudicados con becas y ayudas económicas, se solicita a las carreras los diferentes informes de soporte en cuanto al rendimiento académico de los becarios.

Es importante reconocer el incremento en cuanto a las becas brindadas en la universidad en cada uno de sus componentes como son becas por resultados académicos, becas por situación socioeconómica, becas culturales, becas por discapacidad, y ayudas económicas a estudiantes que asisten en representación de la universidad a eventos científicos nacionales e internacionales, es la suma de esfuerzos y un trabajo conjunto entre la Unidad de Bienestar Estudiantil y las máximas Autoridades.

Sin duda el accionar de la Unidad de Bienestar Estudiantil es importante y a la vez fundamental para poder atender a la comunidad universitaria en cada una de las necesidades que puedan presentar, siempre y cuando se encuentren enmarcadas en los servicios que ofrece la unidad.

\section{Conclusiones}

Las Unidades de Bienestar Estudiantil en las instituciones de Educación superior fueron creadas como una Unidad Administrativa reconocida en la Ley Orgánica de Educación Superior que incluye a dichas unidades como uno de sus pilares estratégicos y fundamentales.

La Universidad Estatal del Sur de Manabí a través de sus autoridades principales se preocupan por el bienestar de la comunidad educativa, asignando recursos económicos a la Unidad de Bienestar Estudiantil que le permita accionar de manera eficiente cada uno de sus procesos.

Se han realizado campañas y divulgación de información para que los estudiantes tengan conocimientos acerca de los beneficios y servicios que brinda la unidad de bienestar estudiantil para ayudar a solucionar problemas y necesidades de la comunidad universitaria.

Los servicios asistenciales han ido mejorando progresivamente, aunque en la actualidad existen aún falencias en cuanto a no contar con médicos especialistas ni una infraestructura adecuada para la atención, se está buscando la ayuda de instituciones de educación superior hermanas que cuentan con mayor presupuesto y mejores prestaciones en la Unidad de Bienestar Estudiantil.

Los resultados mostrados es el accionar de esta unidad, no cabe duda que la meta hay que aún superarla, aumentar esfuerzos y trabajar mancomunadamente con estudiantes, profesores, trabajadores y autoridades.

El contar con esta unidad, da un enfoque positivo al desarrollo de la UNESUM, y gracias a esto garantizan el desarrollo de las capacidades colectivas e individuales que permitan el mejoramiento de la calidad de vida de la comunidad universitaria. 


\section{Referencias bibliográficas}

Asamblea Nacional Constituyente. (2008b). Constitución del Ecuador. Montecristi.

CES. (2010). Ley Orgánica de Educación Superior (LOES) (en línea). http://www.ces.gob.ec/index.php?option $=$ com_phocadownload $\&$ view $=$ category $\&$ id $=11$ :ley-organica-de-educacion superior\&Itemid $=137$.

Rodríguez-Ponce, E. (2009): "El rol de las universidades en la sociedad del conocimiento y en la era de la globalización: Evidencia desde Chile”. Interciencia, 34 (11), 822-829.

Universidad Estatal del Sur de Manabí. (2015). Estatuto UNESUM (en línea). https:/ / drive.google.com/ file/d/0B1yqOTYPrKWPQzBqUGZ6Y0ZmdUU/view, consultado el 6 de enero de 2019.

Universidad Estatal del Sur de Manabí. (26 de octubre de 2015). Reglamentos Generales (en línea). http:// unesum.edu.ec/reglamentosgenerales/reglamento-de_la_unidad_de_bienestar_estudiantil-unesum/, consultado el 10 de enero de 2019.

Universidad Estatal del Sur de Manabí. (2016). Reglamento de la Unidad de Bienestar Estudiantil (en línea). https://drive.google.com/file/d/0B1yqOTYPrKWPQzBqUGZ6Y0ZmdUU/view.

Universidad Estatal del Sur de Manabí. (2019). Reglamento sobre Becas y Ayudas económicas a estudiantes de grado (en línea). https://drive.google.com/file/d/0B1yqOTYPrKWPMGJGZFcwMy1seXc/ view, consultado el 14 de mayo de 2019.

\section{Notas biográficas}

Leopoldo Vinicio Venegas Loor es ingeniero en Computación y Redes, cursó la Maestría en Evaluación y Auditoría de Sistemas Tecnológicos. Es Docente Titular Agregado y Coordinador de la Unidad de Bienestar Estudiantil en la Universidad Estatal del Sur de Manabí. Investiga en el área de las tecnologías de la información y comunicación aplicadas a la educación y cursa estudios de Doctorado en el área de Educación en la Universidad Católica Andrés Bello de Caracas (Venezuela). Está vinculado al proyecto TO-INN.

Paola Yadira Moreira Aguayo es Licenciada en Ciencias de la Educación, especialidad Inglés, cursa la Maestría en Enseñanza del Idioma Inglés. Es Docente Titular Agregado y Coordinadora del Centro de Idiomas de la Universidad Estatal el Sur de Manabí. Investiga en el área de currículo, procesos de enseñanza y aprendizaje en el idioma inglés y cursa estudios de Doctorado en el área de Educación en la Universidad Católica Andrés Bello de Caracas (Venezuela).

Danny Eduardo Chávez Pozo es Licenciada en Ciencias de la Educación, especialización Educación Primaria, cursó la Maestría en Diseños y Evaluación de Modelos Educativos. Investiga en el área de diseño curricular en la educación primaria y secundaria, procesos educativos en la formación universitaria y cursa estudios de Doctorado en el área de Educación en la Universidad Católica Andrés Bello de Caracas (Venezuela). 